\title{
Educação em Saúde da Atenção Primária Brasileira: Uma Pesquisa Documental no Contexto Pandêmico da Covid-19
}

\author{
Débora Santos Lula Barros, ${ }^{1}$ Larissa Otaviano Mesquita, ${ }^{2}$ \\ Tainá Rocha Santos, ${ }^{2}$ Micaela Silva Lopes, ${ }^{2}$ \\ Renata Michele Cassimiro da Silva Oliveira, ${ }^{2}$ Cananda Ferreira Cavalcante ${ }^{2}$
}

\section{RESUMO}

Considerando a educação em saúde como um componente fundamental no processo de cuidado no cenário da pandemia, esta pesquisa objetivou identificar as ações educativas que foram propostas no âmbito da atenção primária à saúde. Para tanto, foi realizada a análise dos documentos produzidos pelas Secretarias de Saúde dos Estados e do Distrito Federal. No geral, esses documentos apresentaram orientações sobre a condução das atividades educativas por meio remoto utilizando diversos mecanismos tecnológicos. Os conteúdos citados com maior frequência corresponderam àqueles voltados à prevenção e ao controle da Covid-19 na comunidade. Os materiais educativos foram relacionados como instrumentos estratégicos na difusão de informações e no combate às fake news. Este estudo contribui para a compreensão da educação em saúde na atenção primária no complexo cenário da pandemia da Covid-19.

Palavras-chave: Educação em saúde; atenção primária à saúde; infecções por coronavírus.

HEALTH EDUCATION IN BRAZILIAN PRIMARY CARE: A DESK RESEARCH IN THE COVID-19 PANDEMIC CONTEXTO

\section{ABSTRACT}

Considering health education as a fundamental component in the care process in the pandemic scenario, this research aimed to identify the educational actions that were proposed in the context of primary health care. To this end, an analysis of the documents produced by the Health Departments of the states and the Federal District was carried out. In general, these documents presented guidelines on how to conduct educational activities remotely using various technological mechanisms. The most frequently cited contents corresponded to those aimed at preventing and controlling Covid-19 in the community. The educational materials were listed as strategic instruments in the dissemination of information and in the fight against fake news. This study contributes to the understanding of health education in primary care in the complex pandemic scenario of COVID-19.

Keywords: Health education; primary health care; coronavirus infections.

RECEBIDO EM: 18/4/2021

MODIFICAÇÕES SOLICITADAS EM: 21/9/2021

ACEITO EM: $15 / 10 / 2021$

\footnotetext{
${ }^{1}$ Autora correspondente. Secretaria de Saúde do Distrito Federal, Brasil. http://lattes.cnpq.br/1459897614268075. https:// orcid.org/0000-0001-6459-7457. debora.farmacia9@gmail.com

2 Fundação Oswaldo Cruz, Brasília, DF- Brasil.
} 


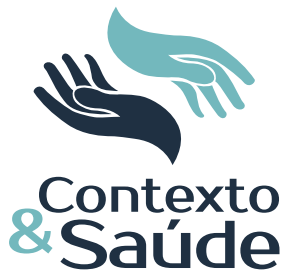

\section{INTRODUÇÃO}

O Sistema Único de Saúde (SUS) conta com estrutura complexa e diversificada, abrangendo diferentes níveis de atenção à saúde, cuja divisão apresenta-se em primária, secundária e terciária. ${ }^{1}$

A Atenção Primária à Saúde (APS), por sua vez, corresponde um conjunto diversificado e complexo de atividades desenvolvidas em contexto multiprofissional. ${ }^{2}$ Ademais, a APS ainda pode ser compreendida como uma política de reorganização do modelo assistencial, quer seja sob forma seletiva ou ampliada, atuando como responsável pela coordenação do cuidado do usuário no SUS. ${ }^{3}$

$\mathrm{Na}$ atual pandemia, a APS desempenha um papel central na mitigação dos efeitos do Coronavirus Disease-2019 (Covid-19), mantendo e aprofundando todos os seus atributos, tais como o acesso ao primeiro contato, a longitudinalidade, a integralidade, a equidade, a universalidade, a coordenação do cuidado, a competência cultural e a orientação familiar/comunitária. ${ }^{4,5}$

A educação em saúde é um dos principais mecanismos para viabilizar a promoção da saúde, a prevenção de doenças e a disseminação de informações aos usuários na pandemia da Covid-19. ${ }^{6}$ Nesse contexto, são fundamentais ações educativas de natureza multidimensional e em caráter interdisciplinar, cuja finalidade é empoderar os usuários de conhecimentos e de habilidades, além de torná-los protagonistas na condução do cuidado em saúde. ${ }^{7-8}$ Assim, para o devido enfretamento da pandemia, algumas reconfigurações foram feitas nos processos de trabalho da APS, uma vez que a condição do distanciamento social resultou na interrupção das atividades coletivas, impactando diretamente na rotina das ações de educação em saúde que são costumeiramente desenvolvidas. ${ }^{9-12}$ Nessa lógica, considerando os desafios impostos para a realização da educação em saúde no atual cenário, este estudo objetivou identificar as orientações que foram propostas pelas Secretarias de Saúde Estaduais e do Distrito Federal sobre o tema da educação em saúde na APS.

\section{METODOLOGIA}

Este estudo trata-se de uma pesquisa documental. Esse percurso metodológico foi adotado para produzir novos conhecimentos, criar novas formas de compreender os fenômenos e entender o modo como estes têm sido desenvolvidos. ${ }^{13}$ Como o objetivo de investigação era a análise das ações propostas pelos documentos governamentais, foi realizado o recrutamento de notas técnicas, notas informativas, notas orientativas, notas recomendatórias, cadernos da atenção primária, guias orientadores, planos de contingência, entre outros, desde que fossem produzidos no período da pandemia pelas Secretarias de Saúde dos Estados e do Distrito Federal. A busca pelos arquivos ocorreu no período de 10 a 28 de janeiro de 2021 nos sites oficiais das secretarias supracitadas. Todos os documentos referentes à pandemia da Covid-19 foram lidos e analisados, com a finalidade de localizar seções textuais que contivessem orientações específicas ao desenvolvimento da educação em saúde na APS. Nesse sentido, na leitura desses documentos foram buscadas as seções que contivessem os Descritores de Ciên-

Editora Unijuí - Revista Contexto \& Saúde - ISSN 2176-7114 - v. 21, n. 44, out./dez. 2021 
cias da Saúde (DECS) sobre a temática e, portanto, além dos termos "educação em saúde" foram considerados como expressões equivalentes: "educar para a saúde", "educação para a saúde", "educação para a saúde comunitária" e "educação sanitária".

Além do mais, alguns documentos apresentavam uma seção genérica sobre educação em saúde na pandemia da Covid-19, mas que por não abordarem instruções específicas para APS, não fizeram parte desta pesquisa documental. Dessa maneira, esse constituiu o único critério de exclusão. Já os critérios de inclusão consistiram os documentos publicados em português até 28 de janeiro de 2021 nos respectivos sites eletrônicos das Secretarias de Saúde dos Estados e do Distrito Federal.

Para o tratamento dos dados foi realizada a análise de conteúdo segundo os pressupostos de Bardin. ${ }^{14}$ Assim, as informações foram sistematizadas de acordo com as seguintes etapas: 1) pré-leitura dos documentos, objetivando a aquisição de uma visão global; 2) leitura seletiva dos documentos, em que se almejou identificar as informações correspondentes ao objetivo da investigação; 3) categorização dos trechos identificados, que foram agrupados em temas de acordo com a semelhança dos conteúdos e 4) análise descritiva e reflexiva dos dados.

Após a análise de conteúdo descrita anteriormente, foram observadas três categorias de discussão que serão debatidas a seguir: 1) Orientações para a realização das atividades de educação em saúde, 2) Conteúdos propostos para as ações de educação em saúde e, por fim, 3) Recomendações para produção e divulgação de materiais educativos.

\section{RESULTADOS E DISCUSSÃO}

A busca realizada por essa investigação encontrou que 15 Estados (62,9\%) publicaram documentos que orientavam como deveria ser desenvolvida a educação em saúde na APS durante a pandemia da Covid-19. Assim, o corpus de análise constituiu-se de 26 documentos técnicos emitidos pelas Secretarias de Saúde das seguintes localidades: Acre, Bahia, Distrito Federal, Espírito Santo, Mato Grosso, Minas Gerais, Pará, Paraná, Tocantins, Rio de Janeiro, Rio Grande do Norte, Rio Grande do Sul, Rondônia, Roraima e Santa Catarina. O Quadro 1 apresenta a caracterização dos documentos analisados.

Quadro 1 - Relação de documentos analisados por Estado brasileiro

\begin{tabular}{|l|l|}
\hline $\begin{array}{l}\text { Secretarias } \\
\text { de Saúde }\end{array}$ & Títulos dos documentos e datas de atualização \\
\hline Acre & $\begin{array}{l}\text { Plano de contingência da Secretaria de Estado de Saúde do Acre para } \\
\text { enfrentamento da infecção pelo novo coronavírus (Covid-19) - atualizado } \\
\text { em junho de } 2020\end{array}$ \\
\hline Bahia & $\begin{array}{l}\text { Plano Estadual de Contingências para Enfrentamento do Novo Coronavírus } \\
\text { - SARS CoV2 - atualizado em março de } 2020\end{array}$ \\
\hline
\end{tabular}

Editora Unijuí - Revista Contexto \& Saúde - ISSN 2176-7114 - v. 21, n. 44, out./dez. 2021 


\begin{tabular}{|c|c|}
\hline \multirow{3}{*}{$\begin{array}{l}\text { Distrito } \\
\text { Federal }\end{array}$} & $\begin{array}{l}\text { Nota Técnica № 01/2020 - Diretrizes de Apoio Clínico e Organizacional } \\
\text { para a APS no enfrentamento à Covid-19 }\end{array}$ \\
\hline & $\begin{array}{l}\text { Nota Técnica SES/SAIS/COAPS - Carteira de Serviços Essenciais para a } \\
\text { Atenção Primária à Saúde durante a pandemia de Covid-19 }\end{array}$ \\
\hline & $\begin{array}{l}\text { Nota Técnica DESF/COAPS/SAIS/SES-DF - Orientações gerais acerca da } \\
\text { retomada das atividades coletivas no âmbito da Atenção Primária à Saúde } \\
\text { no contexto da pandemia (Covid-19) }\end{array}$ \\
\hline $\begin{array}{l}\text { Espírito } \\
\text { Santo }\end{array}$ & $\begin{array}{l}\text { Plano de Contingência do Estado do Espírito Santo para a Infecção } \\
\text { Humana (Covid-19) pelo novo Coronavírus - SARS CoV2 - atualizado em } \\
\text { junho de } 2020\end{array}$ \\
\hline \multirow[b]{2}{*}{$\begin{array}{l}\text { Mato } \\
\text { Grosso }\end{array}$} & $\begin{array}{l}\text { Plano de contingência estadual para infecção humana pelo novo } \\
\text { coronavírus Sars-Cov-2, causador da doença Covid-19 }\end{array}$ \\
\hline & $\begin{array}{l}\text { Nota Técnica Recomendatória № 01/2020 - Recomendação para as } \\
\text { equipes da Atenção Primária (APS) sobre atendimento em situação de } \\
\text { pandemia pela Covid-19 }\end{array}$ \\
\hline \multirow{2}{*}{$\begin{array}{l}\text { Minas } \\
\text { Gerais }\end{array}$} & $\begin{array}{l}\text { Plano de contingência da Secretaria de Estado de Saúde para } \\
\text { enfrentamento do Covid-19 - atualizado em maio de } 2020\end{array}$ \\
\hline & $\begin{array}{l}\text { Guia Orientador da Atenção Primária à Saúde para o Enfrentamento da } \\
\text { Pandemia de Covid-19 }\end{array}$ \\
\hline Pará & $\begin{array}{l}\text { Nota técnica no 001/2020/SESPA: Orientações sobre o processo de } \\
\text { trabalho na Atenção Primária à Saúde }\end{array}$ \\
\hline Paraná & $\begin{array}{l}\text { Plano de contingência do Paraná da Covid-19 (nível } 3 \text { - execução) - } \\
\text { atualizado em junho de } 2020\end{array}$ \\
\hline $\begin{array}{l}\text { Rio de } \\
\text { Janeiro }\end{array}$ & $\begin{array}{l}\text { Plano de contingência da Atenção Primária à Saúde para o coronavírus do } \\
\text { Estado do Rio de Janeiro - atualizado em março de } 2020\end{array}$ \\
\hline \multirow{3}{*}{$\begin{array}{l}\text { Rio Grande } \\
\text { do Norte }\end{array}$} & $\begin{array}{l}\text { Plano de contingência estadual para infecção humana pelo novo } \\
\text { coronavírus - atualizado em dezembro de } 2020\end{array}$ \\
\hline & $\begin{array}{l}\text { Nota Técnica № } 18 / 2020 / \text { SESAP-SUAS/SESAP-CPS/SESAP - Suspender } \\
\text { todas as atividades em grupos (gestantes, hipertensos e diabéticos, } \\
\text { tabagismo, outros) }\end{array}$ \\
\hline & $\begin{array}{l}\text { Caderno da Atenção Primária no Rio Grande do Norte - atualizado em } \\
\text { julho de } 2020\end{array}$ \\
\hline \multirow{4}{*}{$\begin{array}{l}\text { Rio Grande } \\
\text { do Sul }\end{array}$} & $\begin{array}{l}\text { Nota informativa } 24 \mathrm{COE} / \mathrm{SES} \text {-RS: Vigilância de Síndrome Gripal (SG) } \\
\text { e Síndrome Respiratória Aguda Grave (SRAG) relacionada à infecção } \\
\text { humana pela Covid-19, sistemas de notificação, rede laboratorial e } \\
\text { estratégias de testagem - atualizada em outubro de } 2020\end{array}$ \\
\hline & $\begin{array}{l}\text { Nota orientadora para atendimentos odontológicos na Atenção Primária } \\
\text { à Saúde (APS) e Centro de Especialidades Odontológicas (CEO) diante da } \\
\text { epidemia de Covid-19 - atualizada em julho de } 2020\end{array}$ \\
\hline & $\begin{array}{l}\text { Nota de recomendações para a organização das equipes da Atenção } \\
\text { Básica do RS diante da pandemia da Covid-19 -atualizada em janeiro de } \\
2021\end{array}$ \\
\hline & $\begin{array}{l}\text { Recomendações para o trabalho do Agente Comunitário de Saúde no } \\
\text { contexto da pandemia de coronavírus }\end{array}$ \\
\hline Rondônia & $\begin{array}{l}\text { Plano de Contingência do Estado de Rondônia para Medidas de Prevenção } \\
\text { e Controle da Infecção Humana pelo Coronavírus (SARS-CoV-2) - } \\
\text { atualizado em abril de } 2020\end{array}$ \\
\hline Roraima & $\begin{array}{l}\text { Plano de Contingência do Estado de Roraima para enfrentamento da } \\
\text { doença pelo coronavírus } 2019 \text { (Covid-19) - atualizado em março de } 2020\end{array}$ \\
\hline
\end{tabular}




\begin{tabular}{|l|l|}
\hline \multirow{5}{*}{$\begin{array}{l}\text { Santa } \\
\text { Catarina }\end{array}$} & $\begin{array}{l}\text { Plano de Contingência para resposta às emergências em saúde pública - } \\
\text { Doença pelo Sar-Cov-2 (Covid-19) }\end{array}$ \\
\cline { 2 - 3 } & $\begin{array}{l}\text { Nota Técnica no 003/2020 - DAPS/SPS/SES/SC: Recomendações sobre o } \\
\text { trabalho dos Agentes Comunitários de Saúde (ACS) na atenção primária } \\
\text { à saúde para o enfrentamento da pandemia de Covid-19 - atualizada em } \\
\text { abril de 2020 }\end{array}$ \\
\hline \multirow{3}{*}{ Tocantins } & $\begin{array}{l}\text { Plano de Contingência do Tocantins - novo coronavírus (Covid-19) - } \\
\text { atualizado em setembro de 2020 }\end{array}$ \\
\cline { 2 - 3 } & $\begin{array}{l}\text { Nota técnica no 01, de 26 de março de 2020. Orienta a atenção primária } \\
\text { à saúde dos municípios para o enfrentamento do novo coronavírus } \\
\text { (Covid-19) }\end{array}$ \\
\hline
\end{tabular}

Fonte: Elaborada pelos autores.

\section{Orientações para a realização das atividades de educação em saúde}

Em razão do distanciamento social, os documentos deixaram em evidência a necessidade de suspender as atividades coletivas desenvolvidas presencialmente na APS, como, por exemplo, os grupos voltados para educação em saúde de gestantes, tabagistas, pacientes hipertensos, diabéticos, entre outros. $7,8,10,12,15-18$

Os documentos produzidos por algumas Secretarias de Saúde, contudo, recomendaram que a permanência dessas ações estava condicionada à realização por sistema remoto, de modo que o emprego de tecnologias de comunicação pudesse possibilitar o processo educativo dos usuários em domicílio, além de prevenir a ocorrência de aglomerações. ${ }^{7,8,10,12,15-21}$ Logo, uma das medidas recomendadas foi a criação de grupos virtuais para a continuidade das atividades coletivas que foram suspensas. $7,8,10,12,15-17,22$

Embora a utilização da estratégia discutida anteriormente seja fundamental, vale lembrar que é preciso o desenvolvimento de meios alternativos para públicos com dificuldades de acesso/letramento aos dispositivos tecnológicos. ${ }^{10,15-17}$ Assim, foi preconizado que a abordagem comunicativa territorial adotasse amplos dispositivos de comunicação, de forma que seja garantida a disponibilidade de informações para os diferentes grupos da comunidade. ${ }^{10}$ Nesse sentido, alguns documentos propuseram a adoção de diversos canais de comunicação, com destaque para o emprego das rádios comunitárias, bicicletas com caixas de som, jornais comunitários, folhetos/cartazes informativos, programas de televisão, carros de som, grupos de mensagem em aplicativos, informações veiculadas em redes sociais, entre outros. ${ }^{18,23}$

Além das ações coletivas de educação em saúde que podem ser desenvolvidas com os grupos de usuários por meio tecnológico, a modalidade de teleatendimento individual também foi mencionada como ferramenta útil para a educação dos usuários sobre os diversos aspectos que permeiam os cuidados em saúde durante a pandemia. ${ }^{12,22,23}$

Além das ações de educação em saúde por via remota, alguns documentos, produzidos de forma mais recente, orientaram como deveria ser a condução dessas atividades na modalidade presencial. ${ }^{11,24,25}$ Foram citados os atendimen-

Editora Unijuí - Revista Contexto \& Saúde - ISSN 2176-7114 - v. 21, n. 44, out./dez. 2021 


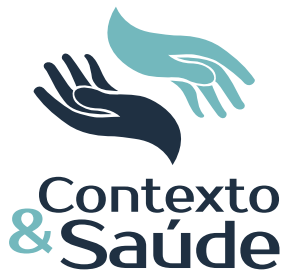
orientada a recomendação de planejar e executar ações educativas em prol de mobilização de lideranças locais, de modo que esses indivíduos fossem empoderados de conhecimentos e, assim, se tornassem agentes multiplicadores de informações baseadas em evidências no que diz respeito ao enfrentamento da Covid-19.7,7,12

Por último, somente um documento analisado destacou a importância da realização de procedimentos avaliativos para estimar a qualidade das atividades educativas. ${ }^{11}$ Desse modo, recomenda-se que para produção e atualização de documentos norteadores da educação em saúde na APS esse aspecto seja amplamente incorporado e valorizado.

\section{Conteúdos propostos para as ações de educação em saúde}

Majoritariamente os documentos consultados destacam a necessidade de realizar atividades educativas sobre temas referentes à doença, com destaque para a discussão dos sinais e sintomas comuns, das formas de transmissão, dos quadros clínicos que requerem ida aos serviços de saúde, dos exames empregados para o diagnóstico, do distanciamento social, das medidas de prevenção da doença e das recomendações de higiene pessoal e ambiental. ${ }^{11,16,17,22,30,32}$

As abordagens das informações supracitadas são fundamentais, contudo a produção do conhecimento deve exceder a perspectiva puramente biomédica das emergências, levando em conta questões micro e macroestruturais dos processos de saúde/doença e suas determinações sociais. .33,34 $^{3}$

Dessa forma, considerando a multicausalidade do processo saúde-doença, alguns documentos recomendaram a criação de espaços coletivos promotores de debate, utilizando a problematização como ferramenta para identificação e 


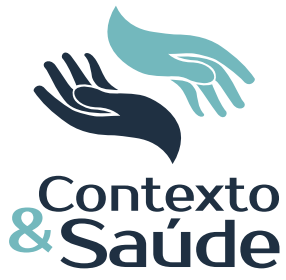

compreensão dos determinantes e condicionantes sociais de saúde presentes na conjuntura atual da pandemia. ${ }^{11,35}$

Na educação em saúde, independentemente do nível de atenção à saúde, uma nova informação deve gerar inquietações e questionamentos sobre as formas de vida adotadas pelos indivíduos. Caracteriza-se, portanto, uma formação que priorize conteúdos capazes de provocar reflexões importantes sobre o cenário de saúde contemporâneo, de modo que sejam ressignificados conceitos e estes estejam orientados para mudanças do contexto de vida, pela indução de uma atitude reflexiva e propositiva dos usuários. ${ }^{7,8}$

Avaliando globalmente os documentos, poucos discutiram que as práticas educativas deveriam abarcar temas voltados ao fortalecimento da autonomia dos indivíduos na gestão dos processos de saúde, achado que não é diferente daquele discutido pelas produções científicas. ${ }^{15}$

A instituição de espaços coletivos de educação em saúde propicia a interação de diferentes atores para a reflexão/ação, de modo que os indivíduos sejam encorajados para a proposição de projetos, tarefas e ações comunitárias em prol da solidariedade e do enfrentamento à Covid-19, colaborando para o ativismo social, o fortalecimento da autonomia e do protagonismo da comunidade adscrita. ${ }^{4}$ Por consequência, alguns documentos orientam que a educação em saúde na APS preveja conteúdos dedicados ao exercício da cidadania voltada para a melhoria das condições de vida, com estímulo à integração e à participação social. $7,8,16,17$

Se a educação em saúde estiver atrelada à perspectiva de capacitações pontuais, de estilo programático, centralizador e com conteúdos padronizados, pode ocorrer o estrangulamento do poder criativo, além de oferecer intervenções que não contemplam a singularidade do território. ${ }^{12,15}$ Por conseguinte, alguns documentos preconizam a indispensabilidade do levantamento dos temas segundo as necessidades educativas da comunidade. ${ }^{11,17}$

É importante destacar que alguns documentos consultados direcionam seu foco de ação para práticas educativas que se inscreveram na temática do autocuidado. ${ }^{12,15}$ Assim, foram sugeridas intervenções educativas com temas voltados a um estilo de vida saudável, incluindo a promoção da alimentação e nutrição adequadas, a prática segura e regular de exercícios físicos, a prevenção do consumo de álcool, tabaco e outras drogas, entre outras orientações. ${ }^{8}$

As Práticas Integrativas e Complementares (PICs), nas últimas décadas, têm recebido incentivo para sua introdução e desenvolvimento na APS. ${ }^{36}$ Nesse sentido, foram recomendadas ações educativas que ofereçam em seus conteúdos instruções em prol da realização das PICs em domicílio, de modo que haja a continuidade no desenvolvimento dessas ações, preservando a integralidade da assistência à saúde e a identidade sociocultural dessa modalidade de cuidado. ${ }^{15,37}$

Em virtude do risco ocupacional e da possibilidade de transmissão da Covid-19 entre os usuários nos consultórios odontológicos da APS, a equipe de saúde bucal apresentou reconfiguração do seu processo de trabalho na pandemia, restringindo o seu exercício laboral, de modo que fossem priorizados os aten-

Editora Unijuí - Revista Contexto \& Saúde - ISSN 2176-7114 - v. 21, n. 44, out./dez. 2021 
dimentos de demandas específicas e emergências. ${ }^{38}$ Nessa conjuntura, alguns documentos reforçam a importância de esses profissionais proverem ações educativas com temas voltados à promoção da saúde bucal da comunidade. 17,20,25,37,39

\section{Recomendações para produção e divulgação de materiais educativos}

Uma das ações propostas pelos documentos emitidos pelas Secretarias de Saúde dos Estados e do Distrito Federal é o emprego de materiais educativos, com ênfase especial para a incorporação de tecnologias comunicacionais inovadoras e criativas, com destaque para a produção de vídeos educativos e cartilhas digitais..$^{40,41}$

A literatura retrata que parte importante dos materiais educativos carrega consigo uma abordagem objetiva com visão estritamente tecnicista, resultando em campanhas e recursos elaborados na perspectiva puramente biomédica. ${ }^{33}$ Para além da tratativa dos conteúdos considerados fundamentais para o compartilhamento de saberes, entretanto, outros recursos e abordagens pedagógicas devem ser empregados na produção do material educativo, adotando linguagens e problematizações diversas, simulando questionamentos produzidos a partir das narrativas da comunidade, acompanhando as especificidades e as singularidades do grupo-alvo da ação educativa. ${ }^{7}$

Alguns documentos analisados enaltecem a importância dos materiais educativos em dirimir os impactos das fake news. ${ }^{24-26}$ Garcia e Duarte ${ }^{42}$ discutem o fenômeno denominado "infodemia", contemporânea da Covid-19. O termo em destaque refere-se a um aumento expressivo do volume de informações associadas à pandemia, que pode se multiplicar exponencialmente em pouco tempo, acarretando rumores e desinformação, além da manipulação de informações com intenção duvidosa.

Outrossim, aos usuários que necessitam de atendimentos no espaço físico das unidades de saúde, além das informações transmitidas verbalmente, foi recomendada a oferta de materiais educativos impressos como reforço da ação educativa. ${ }^{19}$

Por fim, foi citada como oportuna a divulgação de materiais informativos voltados ao enfrentamento da pandemia em espaços sociais da comunidade, tais como igrejas, associações, academias, escolas, entre outros. ${ }^{10,12}$

\section{CONSIDERAÇÕES FINAIS}

De uma forma geral, os documentos apresentam várias possibilidades para o desenvolvimento da educação em saúde na APS no decorrer da pandemia da Covid-19. Recomenda-se priorizar o emprego dos meios remotos para os processos educativos para prevenir aglomerações nos espaços físicos das unidades de saúde ou em outras localidades do território.

Os temas apresentados como indispensáveis nas abordagens educativas corresponderam àqueles dedicados ao controle e à prevenção da Covid-19. Não obstante, outros conteúdos também foram mencionados, tais como: promoção da saúde bucal, da alimentação saudável e da prática regular de exercícios físi-

Editora Unijuí - Revista Contexto \& Saúde - ISSN 2176-7114 - v. 21, n. 44, out./dez. 2021 


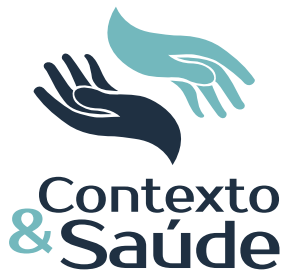

cos; instruções para o desenvolvimento de PIC em domicílio; promoção da autonomia e da autogestão dos indivíduos em saúde e debate dos determinantes sociais da pandemia vigente.

O emprego de materiais educativos foi tratado como essencial para ampliar a comunicação de informações, bem como foi ressaltado o seu papel no combate às fake news.

Este estudo contribui para a compreensão das ações que foram propostas sobre a educação em saúde na APS no complexo cenário da pandemia atual. Para além do conhecimento das ações que foram orientadas pelas Secretarias de Saúde dos Estados e do Distrito Federal, contudo, é crucial o entendimento de como essas informações foram incorporadas nas práticas dos serviços. Nessa esteira, sugere-se que novos estudos superem essa lacuna importante no conhecimento acadêmico.

Uma das limitações da presente pesquisa é a não localização de arquivos nos sites eletrônicos de algumas Secretarias Estaduais de Saúde. Ainda que houvesse uma área para o download de documentos sobre a Covid-19, em algumas pesquisas os arquivos não estavam disponíveis. Vale ressaltar também que em alguns sites o acesso aos arquivos que tratavam da Covid-19 era difícil e confuso. Para exemplificar, alguns sites eletrônicos permitiam a captação de mais de uma versão de um mesmo documento, ainda que somente um arquivo fosse considerado oficial.

\section{REFERÊNCIAS}

1 Arruda C, Lopes SGR, Koerich MHAL, Winck DR, Meirelles BHS, Mello ALSF. Redes de atenção à saúde sob a luz da teoria da complexidade. Escola Anna Nery. 2015;19(1):169-173.

${ }^{2}$ Mendes LV, Campos MR, Chaves GC, Silva RM, Freitas PS, Costa KS et al. Disponibilidade de medicamentos nas unidades básicas de saúde e fatores relacionados: uma abordagem transversal. Saúde Debate. 2014;38(no spe):109-23.

${ }^{3}$ Kleij KS, Tangermann U, Amelung VE, Krauth C. Patients' preferences for primary health care - a systematic literature review of discrete choice experiments. BMC Health Serv Res. 2017;17(1):476.

${ }^{4}$ Daumas RP, Silva GA, Tasca R, Leite IC, Brasil P, Greco DB et al. O papel da atenção primária na rede de atenção à saúde no Brasil: limites e possibilidades no enfrentamento da COVID-19. Cad Saúde Pública. 2020;36: e00104120.

${ }^{5}$ Santos MPA, Nery JS, Goes EF, Silva A, Santos AB, Batista LE, et al. População negra e Covid-19: reflexões sobre racismo e saúde. Estud Av. 2020;34(99):225-44.

${ }^{6}$ Neves VNS, Machado CJDS, Fialho LMF, Sabino RDN. (2021). Utilização de lives como ferramenta de educação em saúde durante a pandemia pela Covid-19. Educ Soc. 42:117.

7 Secretaria de Estado de Saúde de Minas Gerais. Plano de contingência da Secretaria de Estado de Saúde para enfrentamento da Covid-19. 2020. [Acesso em: 27 jan. 2021]. Disponível em: https://coronavirus.saude.mg.gov.br/gestor/profissionais2/plano-de-contingencia

${ }^{8}$ Secretaria de Estado de Saúde de Minas Gerais. Guia Orientador da Atenção Primária à Saúde para o Enfrentamento da Pandemia de Covid-19. 2020. [Acesso em 27 jan. 2021]. Disponível em: https://coronavirus.saude.mg.gov.br/gestor/profissionais2/notas-informativas-alertas-e-outros-documentos

Editora Unijuí - Revista Contexto \& Saúde - ISSN 2176-7114 - v. 21, n. 44, out./dez. 2021 
${ }^{9}$ Maciel FBM, Santos HLPC, Carneiro RAS, Souza EA, Prado NMBL, Teixeira CFS. Agente comunitário de saúde: reflexões sobre o processo de trabalho em saúde em tempos de pandemia de Covid-19. Ciênc Saúde Colet. 2020;25(Suppl 2):4185-95.

${ }^{10}$ Secretaria de Estado de Saúde do Distrito Federal. Nota Técnica SES/SAIS/COAPS - Carteira de Serviços Essenciais para a Atenção Primária à Saúde durante a pandemia de Covid-19. 2020. [Acesso em 28 jan, 2021]. Disponível em: http://www.saude.df.gov.br/ notas-tecnicas/

${ }^{11}$ Secretaria de Estado de Saúde do Distrito Federal. Nota Técnica DESF/COAPS/SAIS/SES-DF - Orientações gerais acerca da retomada das atividades coletivas no âmbito da Atenção Primária à Saúde no contexto da pandemia (Covid-19). 2020. [Acesso em: 28 jan. 2021]. Disponível em: http://www.saude.df.gov.br/notas-tecnicas/

12 Secretaria de Estado de Saúde do Rio de Janeiro. Plano de contingência da atenção primária à saúde para o coronavírus do Estado do Rio de Janeiro. 2020. [Acesso 21 jan. 2021]. Disponível em: https://coronavirus.rj.gov.br/protocolos-e-outras-publicacoes/

${ }^{13}$ Sá-Silva JR, Almeida CD, Guindani JF. Pesquisa documental: pistas teóricas e metodológicas. Revista Brasileira de História \& Ciências Sociais. 2009; 1(1):1-15.

${ }^{14}$ Bardin L. Análise de conteúdo. Lisboa: Almedina; 2009.

${ }^{15}$ Secretaria de Estado de Saúde do Rio Grande do Norte. Plano de contingência estadual para infecção humana pelo novo coronavírus. 2020. [Acesso em: 22 jan. 2021]. Disponível em: https://portalcovid19.saude.rn.gov.br/medidas/planos-de-contingencia/

${ }^{16}$ Secretaria de Estado de Saúde do Rio Grande do Norte. Nota Técnica № 18/2020/ SESAP-SUAS/SESAP-CPS/SESA - Suspender todas as atividades em grupos (gestantes, hipertensos e diabéticos, tabagismo, outros). 2020. [Acesso em: 22 jan. 2021]. Disponível em: https://portalcovid19.saude.rn.gov.br/medidas/

17 Secretaria de Estado de Saúde do Rio Grande do Norte. Caderno da Atenção Primária do Rio Grande do Norte. 2020. [Acesso em: 22 jan. 2021]. Disponível em: https://portalcovid19.saude.rn.gov.br/medidas/

${ }^{18}$ Secretaria de Estado de Saúde do Tocantins. Plano de Contingência do Tocantins - novo coronavírus (COVID-19). 2020. [Acesso em: 18 jan. 2021]. Disponível em: http://integra.saude.to.gov.br/covid19/Publicacoes

${ }^{19}$ Secretaria de Estado de Saúde do Espírito Santo. Plano de Contingência do Estado do Espírito Santo para a Infecção Humana (Covid-19) pelo novo Coronavírus - SARS CoV2. 2020. [Acesso em: 17 jan. 2021]. Disponível em: https://coronavirus.es.gov.br/plano-de-contingencia

${ }^{20}$ Secretaria de Estado de Saúde do Mato Grosso. Plano de contingência estadual para infecção humana pelo novo coronavírus Sars-Cov-2, causador da doença Covid-19. 2020. [Acesso em 23 jan. 2021]. Disponível em: http://www.saude.mt.gov.br/informe/581

${ }^{21}$ Secretaria de Estado de Saúde do Mato Grosso. Nota Técnica Recomendatória № 01/2020 - Recomendação para as equipes da Atenção Primária (APS) sobre atendimento em situação de pandemia pela Covid-19. 2020. [Acesso em: 23 jan. 2021]. Disponível em: http://www.saude.mt.gov.br/informe/581

22 Secretaria de Estado de Saúde do Tocantins. Nota técnica no 01, de 26 de março de 2020. Orienta a atenção primária à saúde dos municípios para o enfrentamento do novo coronavírus (Covid-19). 2020. [Acesso em: 18 jan. 2021]. Disponível em: https:// central.to.gov.br/download/103006

${ }^{23}$ Secretaria de Estado de Saúde do Rio Grande do Sul. Nota de recomendações para a organização das equipes da Atenção Básica do RS frente à pandemia da Covid-19. 2020. [Acesso em: 27 jan. 2021]. Disponível em: https://coronavirus.rs.gov.br/profissionais-da-saude

${ }^{24}$ Secretaria de Estado de Saúde do Distrito Federal. Nota Técnica № 01/2020 - Diretrizes de Apoio Clínico e Organizacional para a APS no enfrentamento à Covid-19. 2020. [Acesso em: 28 jan. 2021]. Disponível em: http://www.saude.df.gov.br/notas-tecnicas/

${ }^{25}$ Secretaria de Estado de Saúde do Rio Grande do Sul. Nota informativa 24 COE/SES-RS: Vigilância de Síndrome Gripal (SG) e Síndrome Respiratória Aguda Grave (SRAG) relacionada à infeç̧ão humana pela Covid-19, sistemas de notificação, rede laboratorial 
e estratégias de testagem. 2020. [Acesso em: 25 jan. 2021]. Disponível em: https:// coronavirus.rs.gov.br/profissionais-da-saude

${ }^{26}$ Secretaria de Estado de Saúde do Acre. Plano de contingência da Secretaria de Estado de Saúde do Acre para enfrentamento da infecção pelo novo coronavírus (Covid-19). 2020. [Acesso em: 10 jan. 2021]. Disponível em: http://covid19.ac.gov.br/planos

${ }^{27}$ Secretaria de Estado de Saúde de Santa Catarina. Nota Técnica no 003/2020 - DAPS/ SPS/SES/SC: Recomendações sobre o trabalho dos Agentes Comunitários de Saúde (ACS) na atenção primária à saúde para o enfrentamento da pandemia de Covid-19. 2020. [Acesso em: 19 jan. 2021]. Disponível em: https://www.saude.sc.gov.br/coronavirus/arquivos/Nota-Tecnica-003-2020-DAPS-SPS-SES-SC.pdf

${ }^{28}$ Secretaria de Estado de Saúde de Santa Catarina. Plano de contingência para resposta às emergências em saúde pública - Doença pelo Sar-Cov-2 (Covid-19). 2020. [Acesso em: 19 jan. 2021]. Disponível em: https://www.saude.sc.gov.br/coronavirus/arquivos/ Plano_contingencia_para_respostas_as_emergencias_em_saude_publica.pdf

${ }^{29}$ Teixeira MG, Medina MG, Costa MN, Barral-Netto M, Carreiro R, Aquino R. Reorganização da atenção primária à saúde para vigilância universal e contenção da Covid-19. Epidemiol Serv Saúde. 2020; 29(4):e2020494.

${ }^{30}$ Secretaria de Estado de Saúde do Paraná. Plano de Contingência do Paraná Covid-19 (nível 3 - execução). 2020. [Acesso em: 19 jan. 2021]. Disponível em: https://www. saude.pr.gov.br/sites/default/arquivos_restritos/files/documento/2020-06/plano_de_ contingencia_do_parana_covid_19_nivel_3_atualizado.pdf

${ }^{31}$ Secretaria de Estado de Saúde do Rio Grande do Sul. Recomendações para o trabaIho do Agente Comunitário de Saúde no contexto da pandemia de coronavírus. 2020. [Acesso em: 27 jan. 2021]. Disponível em: https://coronavirus.rs.gov.br/profissionais-da-saude

${ }^{32}$ Secretaria de Estado de Saúde de Rondônia. Plano de Contingência do Estado de Rondônia para Medidas de Prevenção e Controle da Infecção Humana pelo Coronavírus (SARS-CoV-2). 2020. [Acesso em: 28 jan. 2021]. Disponível em: http://www.rondonia. ro.gov.br/covid-19/institucional/plano-estadual-de-contingencia/

${ }^{33}$ Balbino AC; Silva ANS; Queiroz MVO. O impacto das tecnologias educacionais no ensino de profissionais para o cuidado neonatal. Revista Cuidarte. 2020;11(2):e954.

${ }^{34}$ Ventura DFL, Ribeiro H, Giulio GM, Jaime PC, Nunes J, Bógus CM, et al. Challenges of the Covid-19 pandemic: for a Brazilian research agenda in global health and sustainability. Cad Saude Publica. 2020;36(4):e00040620.

${ }^{35}$ Lima RRT. Formação em gestão do trabalho e da educação na saúde: relatando uma experiência educativa. Saúde Debate. 2020;44(126):919-29.

${ }^{36}$ Tesser CD, Sousa IMC, Nascimento MC. Práticas Integrativas e Complementares na atenção primária à saúde brasileira. Saúde Debate. 2018;42(Suppl 1):174-88.

${ }^{37}$ Secretaria de Estado de Saúde do Rio Grande do Sul. Nota orientadora para atendimentos odontológicos na atenção primária à saúde (APS) e Centro de Especialidades Odontológicas (CEO) frente à Epidemia de Covid-19. 2020. [Acesso em: 27 jan 2021]. Disponível em: https://coronavirus.rs.gov.br/profissionais-da-saude

${ }^{38}$ Carletto AF, Santos FF. A atuação do dentista de família na pandemia da Covid-19: o cenário do Rio de Janeiro. Physis. 2020;30(3):e300310.

${ }^{39}$ Secretaria de Estado de Saúde do Pará. Nota técnica no 001/2020/SESPA: Orientações sobre o processo de trabalho na atenção primária à saúde. 2020. [Acesso em: 17 jan. 2021]. Disponível em: http://www.saude.pa.gov.br/download/nota-tecnica-no-001-2020-orientacoes-sobre-o-manejo-clinico-do-novo-coronavirus-covid-19-e-organizacao-do-processo-de-trabalho-na-atencao-primaria-a-saude-aps-do-estado-do-pa$\mathrm{ra} /$

${ }^{40}$ Secretaria de Estado de Saúde da Bahia. Plano Estadual de Contingências para Enfrentamento do Novo Coronavírus - SARS CoV2. 2020. [Acesso em: 11 jan. 2021]. Disponível em: http://www.saude.ba.gov.br/temasdesaude/coronavirus/plano-estadual-de-contingencias-e-notas-tecnicas-covid-19/ 
${ }^{41}$ Secretaria de Estado de Saúde de Roraima. Plano de contingência do estado de Roraima para enfrentamento da doença pelo coronavírus 2019 (Covid-19). 2020. [Acesso em: 22 jan. 2021]. Disponível em: https://saude.rr.gov.br/index.php/informacoesx/coronavirus/plano-de-contingencia-estadual/file/1053-plano-de-contingencia-estadual-do-coronavirus-2020-2-versao

42 Garcia LP, Duarte E. Infodemia: excesso de quantidade em detrimento da qualidade das informações sobre a Covid-19. Epidemiol Serv Saúde. 2020;29:e2020186.

Financiamento: não houve.

Aspectos éticos: não se aplica.

Conflito de interesses: não há.

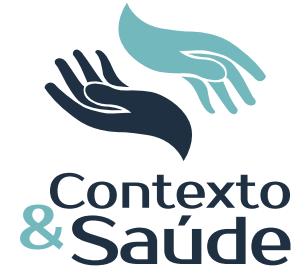

